\title{
Homodinuclear Ferrous Group Metal Complexes and Their Application in Homogeneous Catalysis
}

\author{
WANG Lu ${ }^{1}$, SUN Wei ${ }^{1,2}$, LIU Chao ${ }^{1, *}$ \\ ${ }^{1}$ State Key Laboratory for Oxo Synthesis and Selective Oxidation, Lanzhou Institute of Chemical Physics, Chinese Academy of \\ Sciences, Lanzhou 730000, P. R. China. \\ ${ }^{2}$ University of Chinese Academy of Sciences, Beijing 100049, P. R. China.
}

\begin{abstract}
With the development of synthetic chemistry, more and more efficient catalysts are exploited to activate inert chemical bonds and organic molecules. In the synthetic chemistry, catalysts play an important role, scientists are paying more and more attention to the design and synthesis of catalysts. The majority of catalysts in homogeneous catalytic systems belong to mononuclear active species. In addition to the development of catalysis science, major research is also being conducted on the development of the coordination environment for metal centers to increase their catalytic ability and thereby create enhanced catalytic processes. The catalytic activity of dinuclear catalysts varies from those of mononuclear catalysts. Due to the synergistic effect between the two metal centers, the catalytic activity of dinuclear catalytic

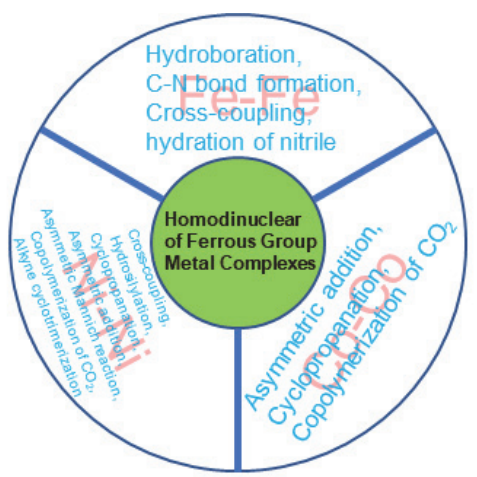
systems exhibits particular performance characteristics. The first row of elements in group VIII of the periodic table, Fe, Co, and Ni, are also known as the ferrous group. These metals have drawn attention in recent years because of their relatively low price, stable structure, commercial availability, and ability to catalyze various type of reactions. Homodinuclear ferrous group metal complexes are applied in a wide variety of reactions, including hydroboration, hydrosilylation, cross-coupling reactions, asymmetric 1,4-addition, asymmetric Mannich reactions, $\mathrm{CO}_{2}$ activation, copolymerization, and alkyne cyclotrimerizations. Compared with mononuclear metal catalytic systems, there are currently relatively few types of homogenous catalytic systems that are catalyzed by bimetal catalysts. However, such catalytic systems possess significant advantages over mononuclear catalytic systems. For example, the catalytic activity and reaction selectivity of dinuclear metal catalytic systems are far superior, the reaction conditions milder, and the operations required simpler. However, research on the mechanisms of dinuclear metal catalytic systems is still insufficient. For example, the interaction between metals and substrates requires further investigation. This review focusses on the synthesis and characterization of homodinuclear bimetallic iron complexes. The application of homodinuclear iron, cobalt, and nickel complexes in homogeneous catalytic systems is introduced and summarized in detail. Finally, the challenges for the future development of homogeneous catalytic systems that utilize homodinuclear bimetallic iron complexes are outlined.
\end{abstract}

Key Words: Homodinuclear complex; Homogeneous catalysis; Iron catalyst; Co catalyst; Ni catalyst

Received: July 28, 2018; Revised: September 4, 2018; Accepted: September 4, 2018; Published online: September 7, 2018.

*Corresponding author. Email: chaoliu@licp.cas.cn; Tel.: +86-13625299463.

The project was supported by the National Natural Science Foundation of China (91745110, 21673261, 21603245, 21633013, 21703265), a Start-up Funding from Lanzhou Institute of Chemical Physics (LICP), the Young Elite Scientist Sponsorship Program by Chain Association for Science and Technology (CAST), the Youth Innovation Promotion Association Chinese Academy of Sciences (CAS) (2018458) and the 'Light of West China' Program. 国家自然科学基金 $(91745110,21673261,21603245,21633013,21703265)$, 中国科学院兰州化学物理研究所启动基金，中国科协 “青年人才托举工程” 项目, 中国科学院青年创新促进会(2018458)以及 “西部之光” 资助项目

(C) Editorial office of Acta Physico-Chimica Sinica 


\title{
同核铁系双金属络合物及其在均相催化体系中的应用
}

\author{
王露 1 , 孙威 1,2 , 刘超 1 ,* \\ 1 中国科学院兰州化学物理研究所, 羰基合成与选择氧化国家重点实验室, 兰州 730000 \\ 2 中国科学院大学, 北京 100049
}

\begin{abstract}
摘要：随着合成化学的不断发展, 开发高活性催化剂来活化一些惰性化学键或者惰性分子受到越来越多的关注。双核金 属络合物作为一类特殊的催化剂展现出了不同于单核金属催化剂的催化活性。在双核过渡金属催化体系中, 因两个金属 中心存在协同作用而表现出了独特的催化活性。铁、钴、镍为第四周期第VIII族元素, 也称为铁系元素。该类金属廉价易 得且参与的催化反应种类繁多, 近年来引起了人们的广泛关注。本综述重点介绍了近年来同核双金属铁系络合物的合成 及其表征。同时，对相关同核铁、钴以及镍催化剂在均相催化体系中的应用也进行了详细的介绍和总结。
\end{abstract}

关键词: 同核双金属催化剂; 均相催化; 铁催化剂; 钴催化剂; 镍催化剂 中图分类号: 0643

\section{1 引言}

在现代有机合成化学中, 金属催化剂具有非常 重要的作用。金属催化剂的种类繁多, 活性区别很 大。随着合成化学的不断发展, 人们正在尝试开发 更多高活性的催化剂来活化一些惰性化学键或者 惰性有机分子。在不断的探索中, 人们发现双核金 属络合物会展现出不同于单核金属催化剂的催化 活性 1,2 。在双核过渡金属催化体系中, 因两个金属 中心存在协同作用而表现出了较好的催化活性 ${ }^{3-6}$ 。 基于双核金属络合物作为催化剂的催化体系研究 目前也取得了一定的进展。其中 $\mathrm{Fe} 、 \mathrm{Co} 、 \mathrm{Ni}$ 为第 四周期第VIII族元素, 统称为 “铁系元素”, 具有 储量丰富、廉价易得、相对安全无毒、环境友好、 生物兼容性好等特点。其次, 铁系金属的金属盐及 其络合物在空气中大多能稳定存在, 且大部分可 直接购买。而且 Fe、Co、Ni既可以实现双电子转 移过程也可以实现单电子转移过程, 这些特性能

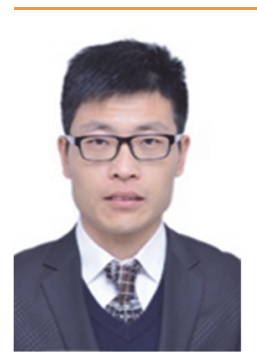

刘超, 2007 年(本科)、2012 年(博士) 毕业于武汉大学化学与分子科学学 院, 师从雷爱文教授, 随后继续在 武汉大学开展博士后研究。博士和 博士后期间在英国杜伦大学 (2008/10-2009/10)、日本分子科学 研究所和名古屋大学(2013/06-08) 短期交流学习。自 2015 年 4 月起加入中国科学院 兰州化物所苏州研究院/羰基合成与选择氧化国家 重点实验室开展独立科研工作。入选中国化学会 “青年人才托举工程” 项目(2016-2018), 荣获 “中 国催化新秀奖” (2017)。主要从事羰基化学、均相催 化研究。
够使得催化剂可以催化多种类型的反应, 因此引 起了人们的广泛关注。目前, 已有较多文献对双核 $\mathrm{Fe} 、 \mathrm{Co} 、 \mathrm{Ni}$ 金属络合物的合成及其性质进行了报 道 ${ }^{7-9}$, 因此本综述主要集中在同核双核铁系金属 络合物在均相催化中的应用, 其中双核羰基金属 络合物不在此综述范围内。

\section{2 双核Fe络合物催化的化学转化}

在2008年, Tatsumi课题组 ${ }^{10}$ 报道了 $\mathrm{N}_{2}$ 桥连的 NHC配位的双核铁络合物 1a (图1), 每一个铁原子 都是典型的三脚钢琴登的构型, 然后通过 $\mathrm{N}_{2}$ 的弱 配位相连接, 同时该络合物是具有抗磁性的。实验 结果表明该络合物可以活化杂环的 $\mathrm{C}-\mathrm{H}$ 键, 但是 目前还不能实现其催化反应。2017年, 山东大学王 文广课题组 ${ }^{11}$ 报道了一例与其结构类似的双核铁 络合物 $\left[\mathrm{Cp}^{*}\left(\mathrm{Ph}_{2} \mathrm{PC}_{6} \mathrm{H}_{4} \mathrm{~S}\right) \mathrm{Fe}\right]_{2}\left(\mu-\mathrm{N}_{2}\right)$ (图1), 该化合 物 $1 \mathrm{~b}$ 是一种中心对称的结构, 两个 $\mathrm{Cp} * \mathrm{Fe}$ $\left(\mathrm{Ph}_{2} \mathrm{PC}_{6} \mathrm{H}_{4} \mathrm{~S}\right)$ 结构由直线型的 $\mathrm{N} \equiv \mathrm{N}$ 连接, $\mathrm{Fe}-\mathrm{N}-\mathrm{N}$ 的键角为 $177.3(4)^{\circ}$ 。通过单晶衍射、拉曼、红外手 段对其结构进行了表征, 两个铁原子中心通过 $\mathrm{N}_{2}$
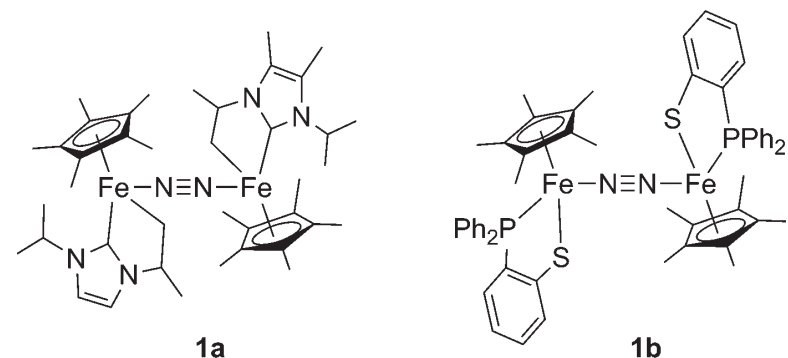

图1 双核铁络合物 $1 \mathrm{a}^{10}$ 和 $1 \mathrm{~b}^{11}$ 的结构比较

Fig. 1 Comparison of the structure of dinuclear iron complexes $1 a^{10}$ and $1 b^{11}$. 
的弱配位相连接。

随后作者考察了络合物 $1 \mathbf{b}$ 对 $N$-杂芳环的硼氢 化反应的活性。结果表明该络合物对含氮杂环类 化合物的硼氢化反应具有很高的活性, 可以在 $50{ }^{\circ} \mathrm{C}$ 甚至室温下高产率高选择性地得到 $N$-喼化的 产物(图2)。该络合物的发现为杂环化合物上引入 喼基团提供了一种新的合成方法与合成思路。随 后, 作者对该反应机理也做出了较为详细的研究 (图3)。在该反应过程中, 络合物 $1 \mathrm{~b}$ 首先会与杂环 的 $\mathrm{N}$ 原子配位生成中间体 $1 \mathrm{c}$, 而后 HBpin上的 $\mathrm{B}$ 与 $\mathrm{S}$ 原子配位生成中间体 $1 \mathbf{d}$, 接着发生 $\mathrm{H}$ 原子的转移过 程, 最终生成目标产物。2013年, Chirik课题组 ${ }^{12}$ 发现了以双亚胺吡啶(PDI型)为配体的, $\mathrm{N}_{2}$ 连接的 双核铁络合物在催化烯烃嗍氢化反应中具有一定 的活性。但是作者在研究中发现, 使用PDI型配体 配位的铁络合物, 无论单核还是双核金属催化剂, 催化活性相差不大。

Betley课题组 ${ }^{13}$ 在 2016 年报道了由两个氯桥连 的双核铁络合物 $\left[\left({ }^{t} \mathrm{BuL}\right) \mathrm{FeCl}\right]_{2}(\mathbf{1 e})$ 。该络合物是一 种绿色的微晶粉末, 可以与芳基叠氮化合物反应, 生成两种新的高自旋Fe(III)络合物。一种是单核 $\mathrm{Fe}-\mathrm{N}$ 络合物 $1 \mathrm{f}$; 另外一种是以氮桥连的双核铁络 合物 $1 \mathrm{~g}$ 。络合物 $1 \mathrm{e}$ 可以活化烯烃 $\alpha$-位的 $\mathrm{C}-\mathrm{H}$ 键和 甲苯苄位 $\mathrm{C}-\mathrm{H}$ 键, 实现 $\mathrm{C}-\mathrm{N}$ 成键反应, 以及苯乙 烯类化合物与芳基叠氮化合物生成氮杂环丙烷的

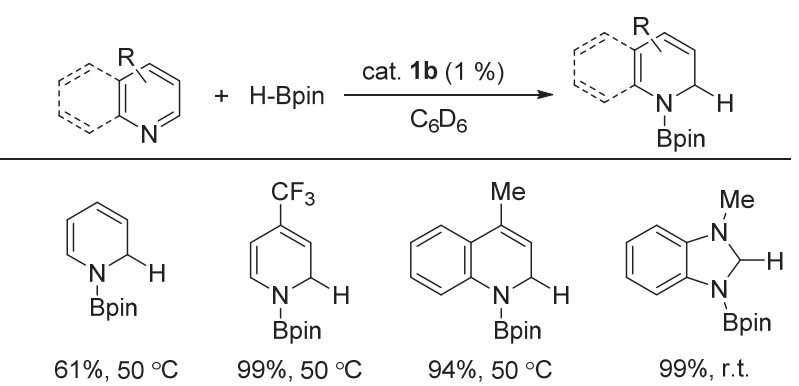

图2 络合物 $1 \mathrm{~b}$ 催化含氮杂环的硼氢化反应 ${ }^{11}$

Fig. 2 1,2-hydroboration of $\mathrm{N}$-heterocycles catalyzed by complex $1 \mathrm{~b}^{11}$.
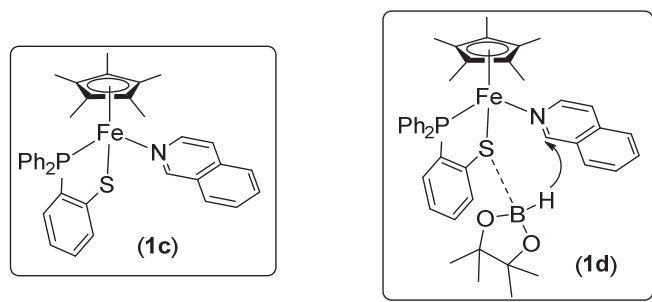

图3 反应中间体以及 $H$ 原子的转移过程 11

Fig. 3 Intermediate of the reaction and $\mathrm{H}$ atom transfer process ${ }^{11}$.

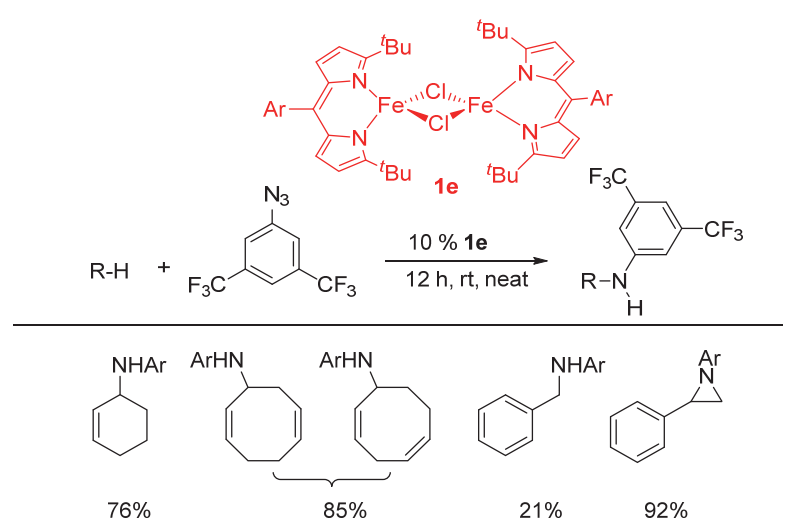

图4 络合物 $1 \mathrm{e}$ 催化的 $\mathrm{C}-\mathrm{N}$ 成键反应 ${ }^{13}$

Fig. $4 \mathrm{C}-\mathrm{N}$ bond formation catalyzed by complex $1 \mathrm{e}^{13}$.

转化(图4)。

在对机理的研究过程中，作者猜测化合物 $\mathbf{1 g}$ 可能是反应的主要中间体, 并且通过 ${ }^{19} \mathrm{~F} \mathrm{NMR}$ 监测 催化剂之间的相互转化(图5)。以芳基叠氮化合物 与环已烯偶联反应为例说明, 首先催化剂 $1 \mathrm{e}$ 与芳 基叠氮化合物反应生成中间体 $1 \mathrm{f}$ 和 $1 \mathrm{~g}, \mathbf{1 f}$ 与芳基叠 氮化合物在催化剂 $1 \mathrm{e}$ 的作用下也会在一定程度上 转化成 $1 \mathrm{~g}, 1 \mathrm{~g}$ 会直接与 $\mathrm{C}-\mathrm{H}$ 化合物发生氢原子的篗 取生成 $1 \mathrm{~h}$ 以及环已烯自由基，1h与环已烯自由基 相互作用生成目标产物，同时也会生成 $1 \mathbf{e}$ 。

2015年, 曲景平课题组 ${ }^{14}$ 报道了以硫负离子桥 连、腈配位的双核 $\mathrm{Fe}$ 络合物(1i)催化腈的水合反应 制备酰胺。在工业生产中, 腈的水合反应都需要在 强酸或者强碱的条件下完成, 条件苛刻, 会产生很 多废物, 不符合绿色化学的理念。早在1994年时,

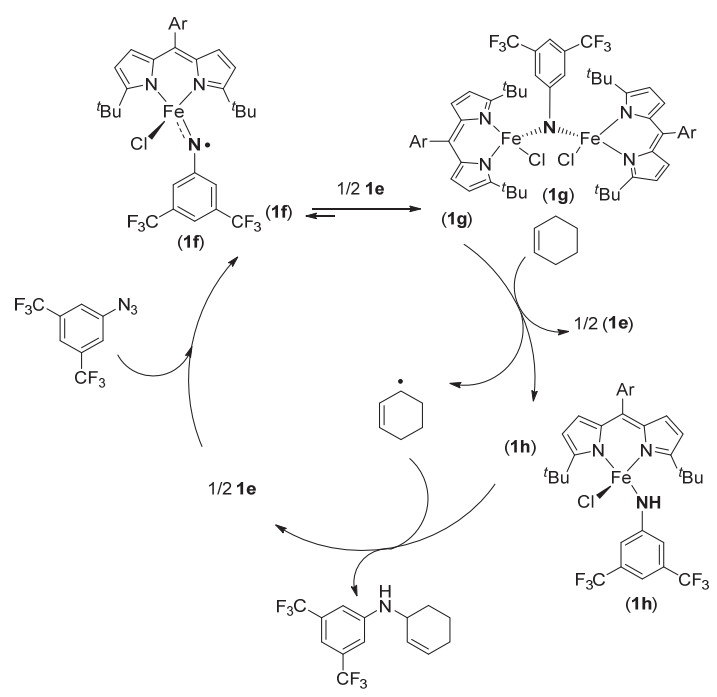

图5 络合物 $1 \mathrm{e}$ 催化 $\mathrm{C}-\mathrm{N}$ 成键的反应机理 ${ }^{13}$

Fig. 5 Mechanism of $\mathrm{C}-\mathrm{N}$ bond formation catalyzed by complex $1 \mathrm{e}^{13}$. 
Que 课题组 ${ }^{15}$ 就报道了一例以氧原子连接的双核 $\mathrm{Fe}$ 促进腈类化合物水解成酰胺, 但是生成的酰胺 的 $\mathrm{N} 、 \mathrm{O}$ 原子分别与双核 $\mathrm{Fe}$ 配位, 形成稳定的五元 环, 无法得到游离的酰胺。曲景平教授通过实验研 究发现, 当向该反应体系中加入 $\mathrm{HBF}_{4} \cdot \mathrm{Et}_{2} \mathrm{O}$ 后, 可 以使其上述与双核铁配位的酰胺解离出来从而得 到游离的酰胺(图6)。该反应体系可以兼容烷基腈、 烯丙基腈、 $N$-氰基类以及芳香腈类化合物, 且可以 在室温下高产率转化成相应的酰胺。

偶联反应在有机合成中有着非常广泛的应 用, 一般都是用活性较高的芳基或者是烯基卤代 物 $(\mathrm{Cl} 、 \mathrm{Br} 、 \mathrm{I})$ 做为亲电试剂。2012年, 邓亮课题 组 ${ }^{16}$ 实现了双核铁络合物催化的非活化一级烷基 氟代物与芳基格氏试剂的偶联反应(图7)。作者通过 与单核铁催化剂相比, 如 $\mathrm{Fe}(\mathrm{acac})_{3} 、\left(\operatorname{IPr}_{2} \mathrm{Me}_{2}\right)$ $\mathrm{Fe}(\mathrm{Mes})_{2}$ 等都几乎得不到目标产物。当使用双核铁 催化剂 $\left(\mathbf{C a t}^{-} \mathbf{F e} \mathbf{e}_{2}\right)$ 时, 却能高效高选择性的得到目 标产物, 反应体系可以兼容各种官能团取代的芳 基格氏试剂以及烷基氟代物。随后的反应机理研 究表明该反应很可能是一个自由基过程(图7)。首 先, 催化剂前体Cat.Fe 在格氏试剂的作用下发生 配体交换, 生成低配位的负离子 $\mathbf{1} \mathbf{j}$ 。而后 $\mathbf{1} \mathbf{j}$ 与 $R F$ 作 用, 发生单电子转移, 产生烷基自由基以及与铁配 位的芳基自由基, 最后这两种自由基偶联后得到 目标产物。

\section{3 双核Co络合物及其催化的化学转化}

从目前的文献报道来看, 双核 $\mathrm{Co}$ 络合物在不

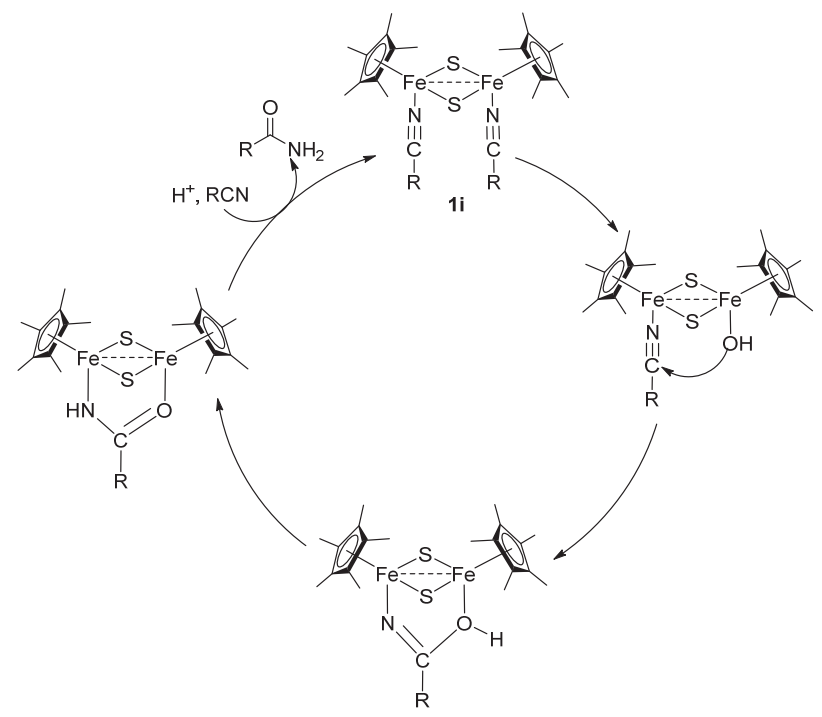

图6 络合物 $1 \mathrm{i}$ 催化的腈水解成酰胺机理 ${ }^{15}$

Fig. 6 Mechanism of hydration of nitrile to amide catalyzed by complex $1 \mathrm{i}^{15}$.

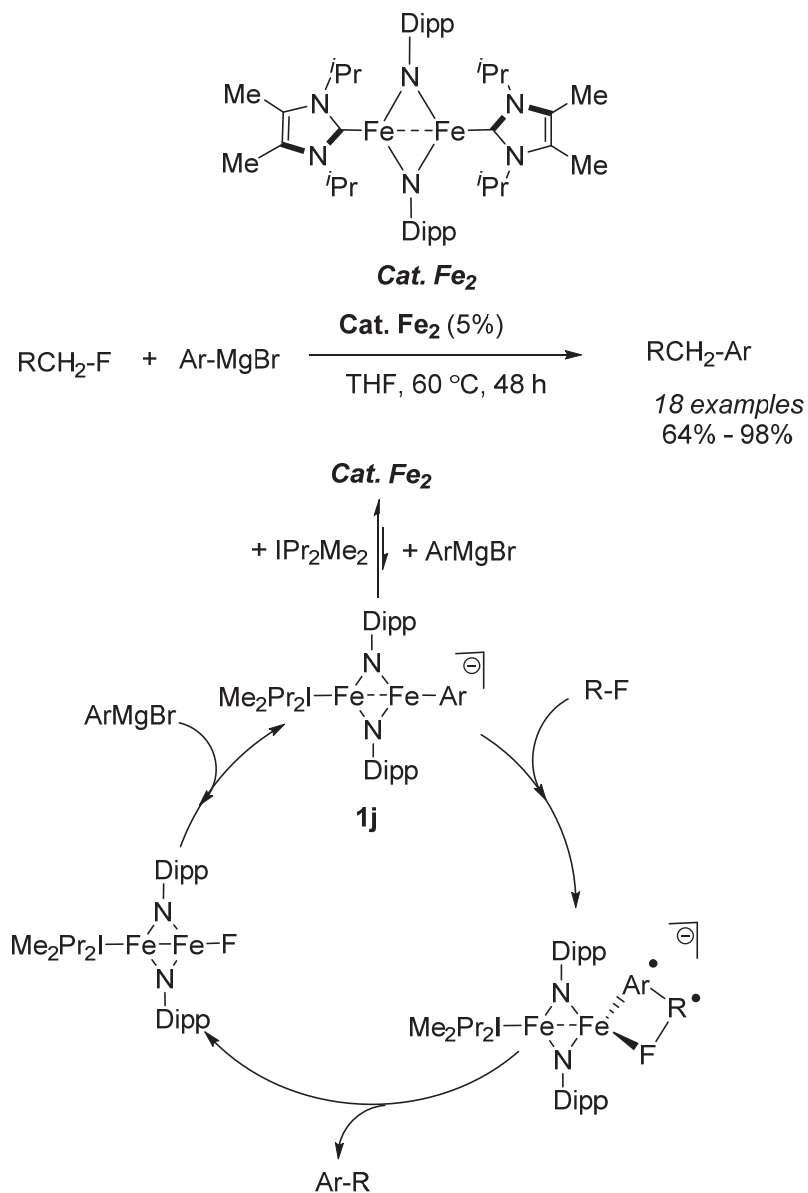

图7 络合物 $1 \mathrm{j}$ 催化的芳基格氏试剂与一级烷基氟代物 偶联反应及其机理 ${ }^{16}$

Fig. 7 Complex $1 \mathrm{j}$ catalyzed cross-coupling of aryl Grignard reagents with primary alkyl fluorides and the proposed mechanism ${ }^{16}$.

对称加成以及 $\mathrm{CO}_{2}$ 催化转化方面应用的较多。

在2009年, Shibasaki课题组 ${ }^{17}$ 报道了席夫碱骨 架的双核 $\mathrm{Co}(\mathrm{III})$ 络合物(R)-Co $\mathbf{C o}_{2}^{\mathrm{III}}(\mathrm{OAc})_{2}-\mathbf{2} \mathbf{a}$ 催化的 $\beta$-酮酸酯与炔酮的不对称 1,4 -加成反应(图8)。该反
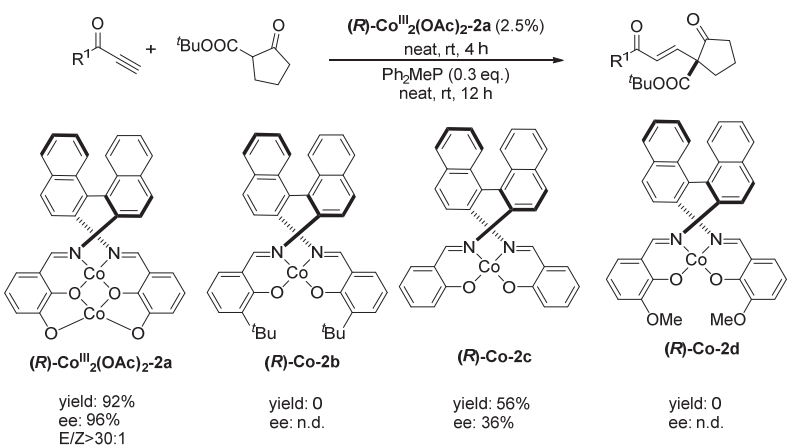

图8 络合物 $(R)-\mathrm{Co}_{2}^{\mathrm{III}}(\mathrm{OAc})_{2}-2 \mathrm{a}$ 催化的 $\beta$-酮酸酯与 炔酮的不对称 $1,4-$ 加成反应 ${ }^{17}$

Fig. 8 Complex (R)-Co ${ }_{2}^{\text {III }}(\mathrm{OAc})_{2}-2$ a catalyzed asymmetric 1,4-addition of $\beta$-keto ester to alkynone ${ }^{17}$. 
应的产率和ee (对映体过量)值都可以达到 $95 \%$ 以 上。这种骨架的双核 $\mathrm{Co}(\mathrm{III})$ 络合物在空气中是稳 定的, 存放六个月以上反应活性都不会降低。而且 在该催化体系中, 催化剂的当量(即用量)可以降低 至 $0.1 \%$ 。作者通过与单核Co金属催化剂的对照, 发 现使用单核Co催化剂时, 选择性和产率都会大大 降低, 甚至根本不能得到目标产物。作者认为两个 Co中心的相互作用对该反应起着很大的作用, 目 前具体作用还不清楚。

在 2005 年, Gao课题组 ${ }^{18}$ 合成了席夫碱骨架的 Robson类型大环配体的双核 $\mathrm{Co}(\mathrm{II})$ 和 $\mathrm{Co}(\mathrm{III})$ 络合 物, 用于苯乙烯与重氮乙酸乙酯的不对称环丙烷 化反应(图9)。在研究过程中, 作者发现, 两种配 体 L1 和 L2 组成的二价和三价 L1-Co(II)、L1$\mathrm{Co}(\mathrm{III}) 、 \mathrm{~L} 2-\mathrm{Co}(\mathrm{II}) 、 \mathrm{~L} 2-\mathrm{Co}(\mathrm{III})$ 钴络合物, 在催化该 反应时, 产率、ee值以及反式/顺式环丙烷的比例 都很接近, 说明这几种络合物催化该反应的机理 是一样的。在该反应过程中, 会产生两个金属-卡 宾中间体。由于这两个卡宾会相互作用, 导致这两 个卡宾重排, 成平行的位置, 而这两个金属卡宾与 络合物的平面呈垂直状态。这样的位置降低酯之 间的空间位阻, 以及与轴向氢原子间的相互作用。

过渡金属催化的 $\mathrm{CO}_{2}$ 与环氧乙烷类化合物制 备环状碳酸酯以及聚碳酸酯是非常重要的一类反 应。该类反应能有效的利用 $\mathrm{CO}_{2}$ 合成大分子量且可 以生物降解的聚合物(图 10)。Williams课题组 ${ }^{19}$ 发 展了两种双核钴 $(\mathrm{Co}(\mathrm{II} / \mathrm{II})$ 和 $\mathrm{Co}(\mathrm{II} / \mathrm{III})$ 络合物, 在 1 个大气压, $80{ }^{\circ} \mathrm{C}$ 的条件下完成了环氧乙烷与 $\mathrm{CO}_{2}$ 的聚合反应。当使用络合物 $\mathbf{2} \mathbf{f}$ 时, TON (turnover number, 单位活性位转化的底物分子数) 可以达到 420, TOF (turnover frequency, 单位时间单位活化位 转化的底物分子数)达到 $159 \mathrm{~h}^{-1}$, 而分子量最高可

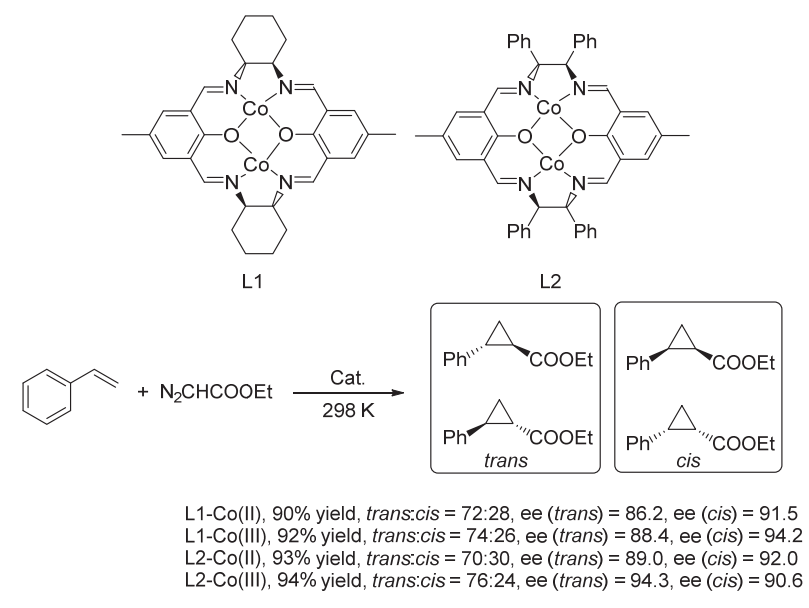

图9 双核 Co催化的环丙烷化反应 ${ }^{18}$

Fig. 9 Dimetallic Co catalyzed cyclopropanation ${ }^{18}$.
以达到 6300 。但是该反应还存在一定的缺陷, 聚合 物分子量比较小, 可能是由于链转移反应的影响。

在以往的催化体系中, 都会观测到环氧乙烷 自身聚合的副产物, 且分子量也比较小。为了解决 这一问题, Ko 课题组 ${ }^{20}$ 于 2016 年合成了以双苯并 三唑亚胺酚类为基本骨架的双核 $\mathrm{Ni} 、 \mathrm{Co}$ 络合物, 并 将其应用到环已烯氧化物与 $\mathrm{CO}_{2}$ 的反应中(图11)。研 究发现双核Co络合物 (2g) 以及双核 $\mathrm{Ni}(\mathbf{2 h})$ 展现出 很好的催化活性, 聚合的选择性可以达到 $99 \%$ 以 上。特别是络合物 $\mathbf{2 h}, 24 \mathrm{~h}$ 时 TON $>1100$, 聚甲基 乙撑碳酸酯 $(\mathrm{PCHC})$ /环己烯碳酸酯 $(\mathrm{CHC})$ 的选择性 也在 $99 / 1$ 以上, 且催化剂的当量可以降低至 $0.03125 \%$ 。当反应温度升高至 $150^{\circ} \mathrm{C}$ 时, 催化剂 $\mathbf{2 h}$ 仍然可以稳定存在, 并且保持很高的反应活性。当

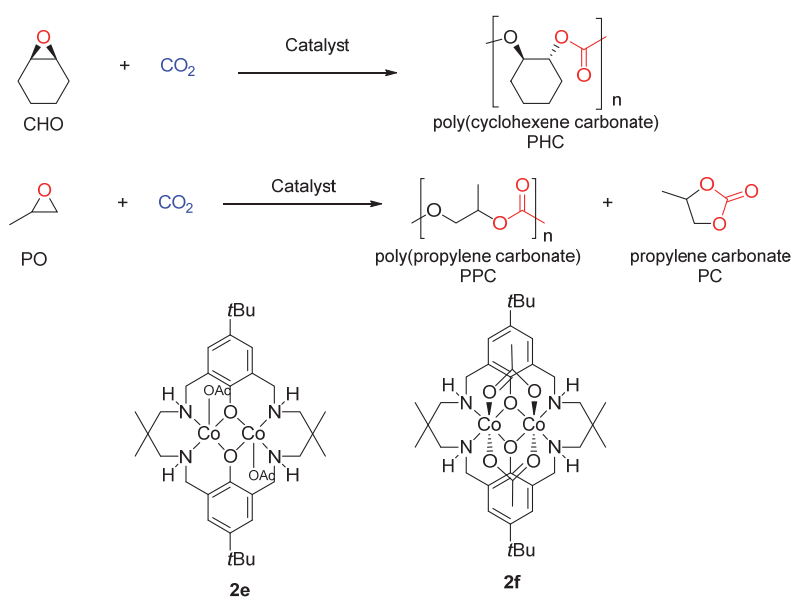

图10 双核 $\mathrm{Co}$ 催化的环氧乙烷类化合物与

$\mathrm{CO}_{2}$ 的聚合反应 ${ }^{19}$

Fig. 10 Copolymerization of $\mathrm{CO}_{2}$ with ethylene oxide compounds catalyzed by dimetallic $\mathrm{Co}^{19}$.

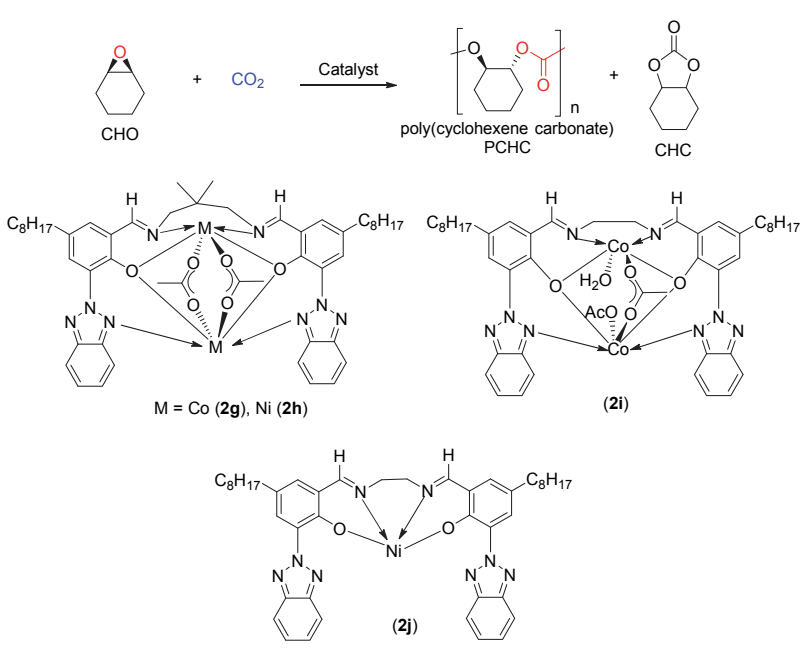

图11 双核 $\mathrm{Co}$ 催化的环己烯氧化物与 $\mathrm{CO}_{2}$ 的聚合 ${ }^{20}$

Fig. 11 Copolymerization of cyclohexene oxide and $\mathrm{CO}_{2}$ catalyzed by dimetallic $\mathrm{Co}^{20}$. 
具有相同配体骨架的单核 $\mathrm{Ni}$ 催化剂 $\mathbf{2} \mathbf{j}$ 时, 该聚合反 应基本不进行。当 $\mathrm{Bu}_{4} \mathrm{NX}(\mathrm{X}=\mathrm{Cl}, \mathrm{Br}, \mathrm{I})$ 作为共催 化剂时, 反应的选择性完全发生改变, 环己烯碳酸 酯 $(\mathrm{CHC})$ 成为主产物, 双核Co (2g)的活性最高, 选 择性在 $99 \%$ 以上, 几乎观测不到聚合的产物生成。

二胺类型(DiBTP)的骨架比亚胺类型(BiIBTP) 骨架的灵活性更强, 2016年Lin课题组 ${ }^{21}$ 报道了 DiBTP类型的双核 Ni络合物 (图 12)对环已烯氧化 物 $(\mathrm{CHO})$ 与 $\mathrm{CO}_{2}$ 的共聚具有很好的催化活性, TON 值可以达到 4000 以上, TOF值也可以到 $400 \mathrm{~h}^{-1}$ 。同 时, 这类催化剂还可以催化乙烯基取代的环己烯 氧化物与 $\mathrm{CO}_{2}$ 的聚合反应, 且分子量可以达到 41800 。

\section{4 双核 Ni络合物及其催化的化学转化}

基于席夫碱骨架的双核镍络合物是一种很稳 定的络合物, 该催化剂在不对称催化反应中有着

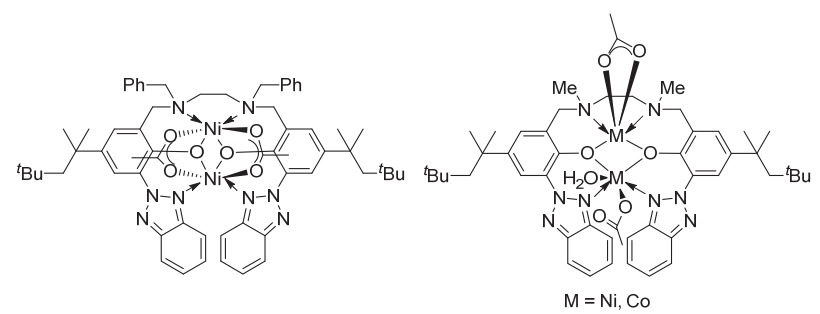

图12 DiBTP类型的双核 $\mathrm{Ni} 、 \mathrm{Co}$ 络合物 ${ }^{21}$

Fig. 12 Bimetallic Ni and Co complexes containing the DiBTP ligand backbone ${ }^{21}$.

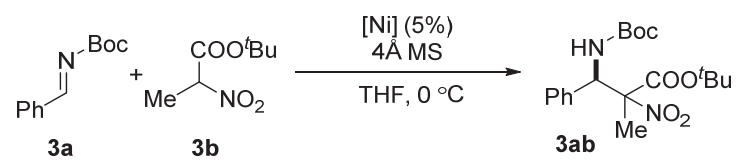

$\begin{array}{cl}(\boldsymbol{R})-\mathbf{N i}_{2}-\mathbf{3 c} & 95 \% \text { yield, } 91: 9 \mathrm{dr}, 98 \% \text { ee } \\ \mathbf{3 c} & 38 \% \text { yield, } 77: 23 \mathrm{dr}, 57 \% \text { ee } \\ \mathbf{3 d} & 22 \% \text { yield, } 57: 43 \mathrm{dr}, 4 \% \text { ee } \\ \mathbf{3 e} & 17 \% \text { yield, } 68: 32 \mathrm{dr}, 3 \% \text { ee } \\ \mathbf{3 f} & 58 \% \text { yield, } 77: 23 \mathrm{dr}, 2 \% \text { ee }\end{array}$

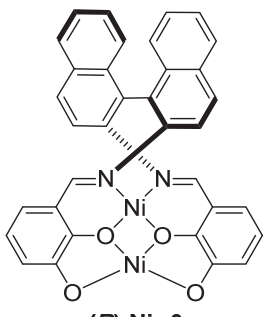

$(R)-\mathrm{Ni}_{2}-3 \mathrm{C}$

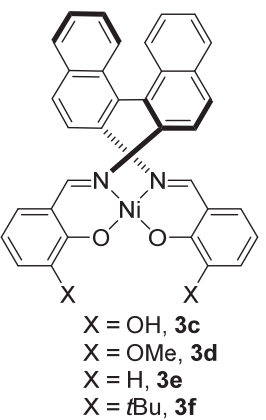

图13 双核 $\mathrm{Ni}$ 与单核 $\mathrm{Ni}$ 催化剂的催化活性对比 ${ }^{22}$

Fig. 13 Comparison the catalytic activity of bimetallic $\mathrm{Ni}$ with mononuclear $\mathrm{Ni}^{22}$.
广泛的应用。

2008年, Shibasaki和Matsunaga课题组 22 报道了 基于席夫碱骨架的双核 $\mathrm{Ni}$ 络合物 $(\boldsymbol{R})-\mathrm{Ni}_{2}-\mathbf{3 c}$ 催化的 不对称Mannich加成反应合成具有手性的 $\alpha, \beta$-二氨 基羧酸衍生物(图13)。这种双核 $\mathrm{Ni}$ 络合物可以在空 气中稳定存放 3 个月, 且催化活性不会降低, 当使用 3a、3b作为反应底物时, 能以 $95 \%$ 的收率, $91: 9$ 的 $\operatorname{dr}$ (非对映比)值以及 $98 \%$ 的ee值得到目标产物。该金 属催化剂与席夫碱骨架的单核 $\mathrm{Ni}$ 络合物的反应活 性相差较大。当使用单核 $\mathrm{Ni}$ 作为催化剂时, 反应活 性、产率和选择性都有所降低。由此说明, 两个金 属 $\mathrm{Ni}$ 中心的相互作用对反应选择性以及产率都有 较大的影响, 目前该作用还不清楚, 还在进一步的 研究中。

同年, 该课题组使用 $\beta$-酮磷酸酯时, 也能获得 理想的产率与选择性(图 14, 方程式 1$)^{23}$ 。作者猜测 这种双核金属 $\mathrm{Ni}$-芳基氧的部分是起到Brønsted碱 的作用, $\beta$-酮磷酸酯烯醇式中的氧原子以及磷酸 酯上的氧与其中一个 $\mathrm{Ni}$ 中心配位, $N$-Boc亚胺中的 羰基氧会与另一个 $\mathrm{Ni}$ 中心配位(图 14)。最终以 $90 \%$ 产率, $20: 1$ 的 dr值, $99 \%$ 的ee值得到反式加成的产 物。随后, 他们继续使用该催化剂在室温下完成了 亚胺或者硝基取代的烯烃类化合物对 $\alpha, \beta$-不饱和$\gamma$-丁内酰胺的不对称加成反应(图 14, 方程式2-3),
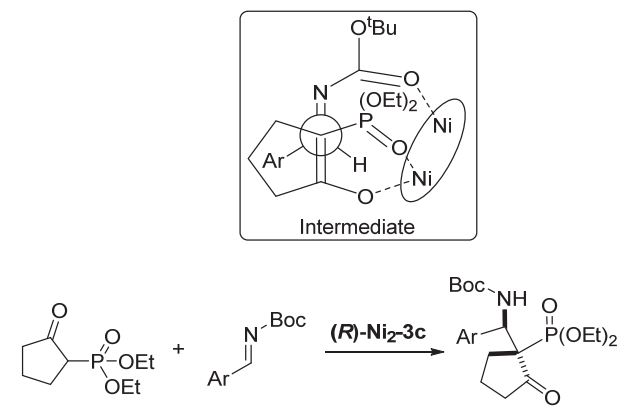

(1)

$43 \%-90 \%$ yield, $2: 1-20: 1 \mathrm{~d}$ $84 \%-99 \%$ ee

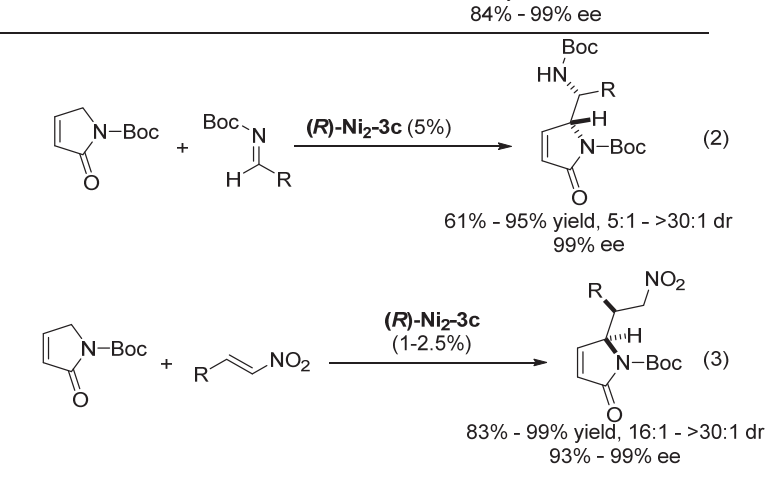

图14 络合物 $(R)-\mathrm{Ni}_{2}-3 \mathrm{c}$ 催化的不对称加成以及 反应中间体的猜测 23,24

Fig. 14 Complex $(R)-\mathrm{Ni}_{2}-3 \mathrm{c}$ catalytic asymmetric addition reaction and the proposed intermediate ${ }^{23,24}$. 
dr值可以达到 $30: 1$, ee值高达 $99 \%$, 而以席夫碱为 基本骨架的单核 $\mathrm{Ni}$ 金属催化剂是没有活性的, 即 使反应时间延长至 $24 \mathrm{~h}$ 也检测不到目标产物 ${ }^{24}$ 。

该课题组发现该催化剂对于 $\beta$-酮酸酯与甲醛 的不对称Aldol缩合反应也具有很高催化活性 ${ }^{25}$, 其 中催化剂的当量可以降低至 $0.1 \%$, 在较温和的条 件下最高可以达到 $95 \%$ 的ee值。除此之外, 该类催 化剂还被他们用于催化 1,1 -二磷酸酯烯烃的不对 称 1,4 -加成反应 ${ }^{26}$ 。随后, 他们将这类催化剂用于 $\alpha$-酮酰胺与不饱和 $\beta$-酮酸酯和硝基化合物的不对 称反应 ${ }^{27,28}$ 。他们在文章中提出, $\alpha$-酮酰胺与不饱 和 $\beta$-酮酸酯先形成烯醇结构, 双核镍催化剂中的 两个金属中心分别与两种底物作用, 一个 $\mathrm{Ni}$ 中心 与烯醇结构配位, 另一个 $\mathrm{Ni}$ 中心作为Lewis酸与硝 基取代的烯烃配位, 最后两分子发生加成-质子化 作用得到目标产物。

2012年, Shibasaki课题组 ${ }^{29}$ 报道了该结构的双 核镍催化剂还可以催化3-取代戊二酸酐的开环酯 化反应, 在较温和的条件下就得到 $\gamma$-酸酯类化合物 (图15)。当使用 $\mathrm{S}$ 构型的催化剂前体 $(S)-\mathrm{Ni}_{2}-3 \mathrm{c}$ 时, 得 到的目标产物也会发生构型翻转, ee值保持不变。

2017年, 周辉课题组 ${ }^{30}$ 报道了含有磺酰胺结构 的NON配位的双核Ni (3g)催化的不对称曼尼希反 应(图16)。该反应的催化剂当量可以降低至 $0.05 \%$,

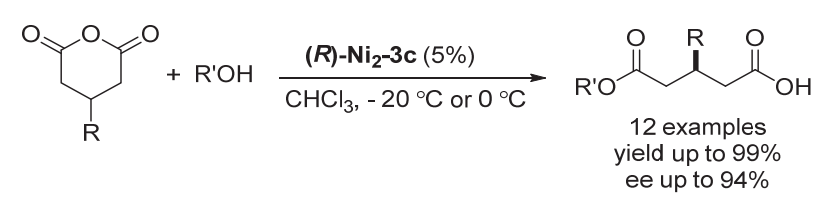

图15 络合物 $(R)-\mathrm{Ni}_{2}-3 \mathrm{c}$ 催化3-取代戊二酸䣶的 去不对称化反应 ${ }^{29}$

Fig. 15 The desymmetrization of 3-substituted glutaric anhydrides catalyzed by complex $(R)-\mathrm{Ni}_{2}-3 \mathrm{c}^{29}$.

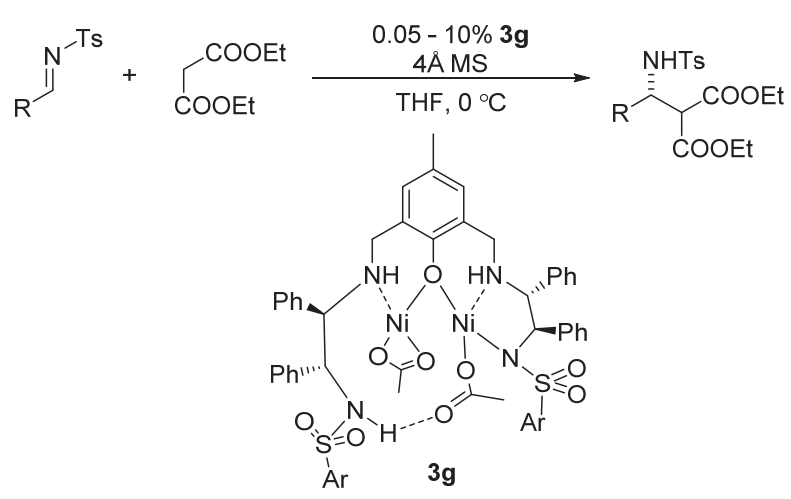

图16 络合物 $3 g$ 催化的不对称曼尼希反应 ${ }^{30}$

Fig. 16 The asymmetric Mannich reaction catalyzed by complex $3 \mathrm{~g}^{30}$. 而产率和ee值基本上不会发生改变, 反应可以扩 大至 $4 \mathrm{~g}$ 以上。

除了席夫碱骨架的双核 $\mathrm{Ni}$ 具有很好的催化活 性外, NHC配位的具有 $\mathrm{Ni}-\mathrm{Ni}$ 键的双核 $\mathrm{Ni}$ 络合物 在催化化学转化时也具有很好的活性。2003年, Sigman课题组 ${ }^{31}$ 报道了 $\mathrm{NHC}$ 配位的单齿 $\pi$-烯丙基 $\mathrm{Ni}(\mathrm{II})$ 络合物催化对氧气具有一定的活化能力。该 络合物是 $\mathrm{Ni}(\mathrm{COD})_{2}$ 与烯丙基类化合物 (烯丙基氯 或者肉桂基氯)、卡宾配体合成的 $\pi$-烯丙基 $\mathrm{Ni}$ (II)$\mathrm{NHC}$, 该络合物可以被氧气氧化成O-桥连的 $\mathrm{Ni}(\mathrm{II})$ 络合物。但是当使用 $\mathrm{Ni}(\mathrm{COD})_{2}$ 与4-氯-2,4-二甲基2-戊烯反应时, 却得到了一些混合物, 最终以 $10 \%-$ $20 \%$ 的收率分离得到了单齿卡宾配位的具有 $\mathrm{Ni}-\mathrm{Ni}$ 键的氯桥联的双核 $\mathrm{Ni}(\mathrm{I})$ 络合物, 这种中性 单齿配体配位的 $\mathrm{Ni}$ 络合物是比较少见的。接着作 者通过 $\mathrm{Ni}(\mathrm{COD})_{2}$ 与 $\mathrm{NiCl}_{2}(\mathrm{DME})$ 在卡宾配体的作用 下, 可以以很高产率得到这种络合物(图17)。

2009年, Hillhouse课题组 ${ }^{32}$ 报道了与该结构类 似的双核 $\mathrm{Ni}$ 络合物, $[(\mathrm{IPr}) \mathrm{Ni}(\mu-\mathrm{Cl})]_{2}(\mathbf{3 h})$ 。[(IPr) Ni $(\mu-\mathrm{Cl})]_{2}$ 与 $2,4,6$-三甲基苯的叠氮化合物反应生成 $d_{8}-d_{8}$ 酰亚胺 Ni的络合物 $\left[(\mathrm{IPr}) \mathrm{NiCl}_{2}(\mu-\mathrm{NMes})(\mathbf{3 i})\right.$, 使 用 $\mathrm{NaBArF}$ 擢取氯原子后得到 $d_{8}-d_{8}$ 二聚体 $\left[\{(\mathrm{IPr}) \mathrm{Ni}\}_{2}(\mu-\mathrm{Cl})(\mu-\mathrm{NMes})\right] \mathrm{BArF}_{4}(\mathbf{3 k}) 。\{(\mathrm{IPr}) \mathrm{NiCl}\}_{2}$ $\left(\mu\right.$-NMes) 可以被 $\mathrm{KC}_{8}$ 还原成 $d_{8}-d_{9}$ 混合价态的 $\{(\mathrm{IPr}) \mathrm{Ni}\}_{2}(\mu-\mathrm{Cl})(\mu$-NMes $)(3 \mathrm{l})$ 络合物。\{(IPr)NiCl $\}_{2}$ $\left(\mu\right.$-NMes) (3i) 可以发生氮烯转移到 $\mathrm{CO}, \mathrm{PMe}_{3}$ 以及 $\mathrm{CNR}\left(\mathrm{R}=\mathrm{PhCH}_{2}, \mathrm{CMe}_{3}\right)$ 的过程(图 18)。最终, Hillhouse 课题组把 $\{(\mathrm{IPr}) \mathrm{Ni}(\mu-\mathrm{Cl})\}_{2}$ (3h) 以及 $\left[\{(\mathrm{IPr}) \mathrm{Ni}\}_{2}(\mu-\mathrm{Cl})(\mu-\mathrm{NMes}) \mathrm{BArF}_{4}(\mathbf{3 k})\right.$ 用于催化有 机叠氮化合物的氮烯转化合成一系列的碳化二亚 胺、异氘酸酯类的反应中(图19)。

这类以卡宾为配体的, 卤素桥连的双核金属 $\mathrm{Ni}$ 催化剂在催化偶联反应中也具有很重要的应用 价值, 较为常见的应用有Kumada-Tamao-Corrious

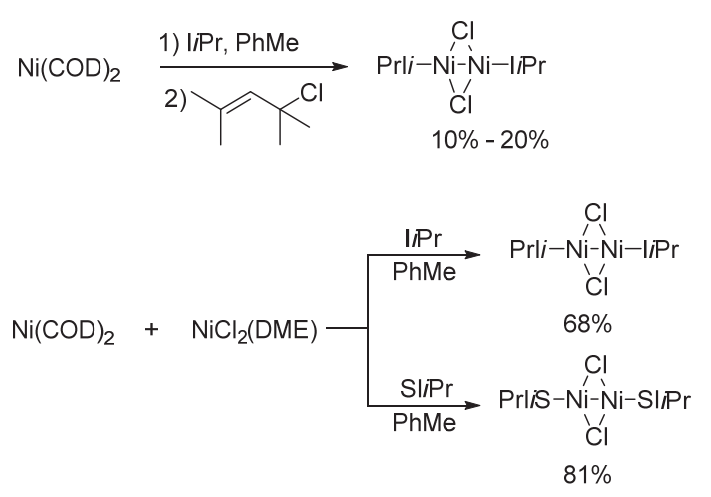

图17 氯桥联的双核 Ni络合物的合成 ${ }^{31}$

Fig. 17 Synthesis of bimetallic Ni complexes ${ }^{31}$. 


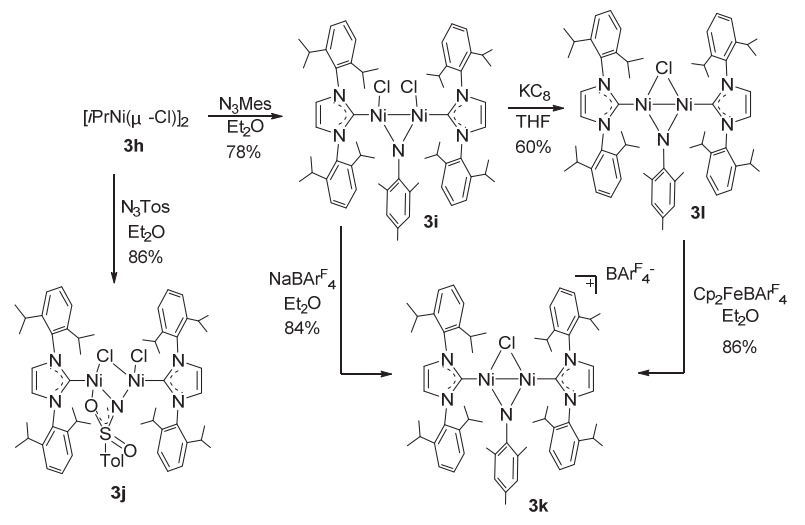

图18 $\{(\mathrm{NHC}) \mathrm{Ni}(\mu-\mathrm{Cl})\}_{2}$ 络合物的转化 ${ }^{32}$

Fig. 18 The transformation of complex $\{(\mathrm{NHC}) \mathrm{Ni}(\mu-\mathrm{Cl})\}_{2}{ }^{32}$.

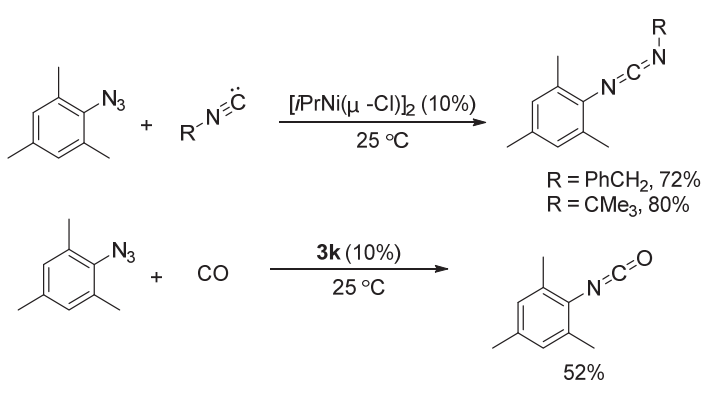

图19 双核Ni催化的有机叠氮化合物的转化 ${ }^{32}$

Fig. 19 The conversion of organoazides catalyzed by bimetallic Ni complex ${ }^{32}$.

偶 联 ${ }^{33,34}$, Buchwald-Hartwig 胺化 ${ }^{35}$, SuzukiMiyaura偶联 ${ }^{36}$ 以及芳基碘代物的三氟甲硒基化反 应 ${ }^{37}$ (图20)。

除了以卡宾为配体的双核金属 $\mathrm{Ni}$ 可以催化偶 联反应外, 2014年, 王中夏课题组 ${ }^{38}$ 报道了 N配体 的单核 $\mathrm{Ni}$ 与双核 $\mathrm{Ni}$ 催化的芳基卤代物 (拟卤代物) 与锌试剂或者格氏试剂的偶联反应(图21)。通过底 物扩展以及与单核 $\mathrm{Ni}$ 催化剂的对比发现, 双核 $\mathrm{Ni}$ 催化剂的兼容性更好, 反应活性也更高一些, 有的 几乎能得到当量转化的产物。主要是由于双核金 属络合物的两个金属中心分别会与芳基卤代物的 芳基和 $\mathrm{C}-\mathrm{X}$ 键作用，这种协同作用有利于金属与 芳基卤代物的氧化加成。

2014年, Uyeda课题组 ${ }^{39}$ 合成了一种基于 1,8䒬啶 -二亚胺结构 (NDI) 的双核 $\mathrm{Ni}$ 络合物 $\left({ }^{i \mathrm{Pr}} \mathrm{NDI}\right) \mathrm{Ni}_{2}\left(\mathrm{C}_{6} \mathrm{H}_{6}\right)(\mathbf{3 n})$ 。该催化剂可以发生多电子 的氧化还原过程, 有五种不同的氧化态(图22), 可 以催化许多类型的化学转化。

由于该双核金属 $\mathrm{Ni}$ 络合物具有多种氧化还原 态, Uyeda课题组将其应用于各种催化反应中。2015 年, Uyeda课题组 40 发现该金属催化剂可以催化烯
烃、炔烃、醛、酮、酰胺等化合物的硅氢化反应, 都能以很高产率得到目标产物(图23)。随后也与单 核金属 $\mathrm{Ni}$ 催化进行了比较, 发现催化剂3n比单核 催化剂3o 和 $3 p$ 的效率高很多, 3o 和 $3 p$ 几乎不能催 化二苯乙炔的硅氢化反应。在反应过程中, 该双 核金属 $\mathrm{Ni}$ 络合物可以很快的与 $\mathrm{Ph}_{2} \mathrm{SiH}_{2} 、 \mathrm{Et}_{2} \mathrm{SiH}_{2}$ 反 应生成络合物。通过单晶衍射, 发现在该配位过 程中, $\mathrm{H}-\mathrm{Si}-\mathrm{H}$ 的键角以及 $\mathrm{Si}-\mathrm{H}$ 键的键长都发生 了较大的改变。而NDI中的 $\pi$-电子有利于该过程的 进行。

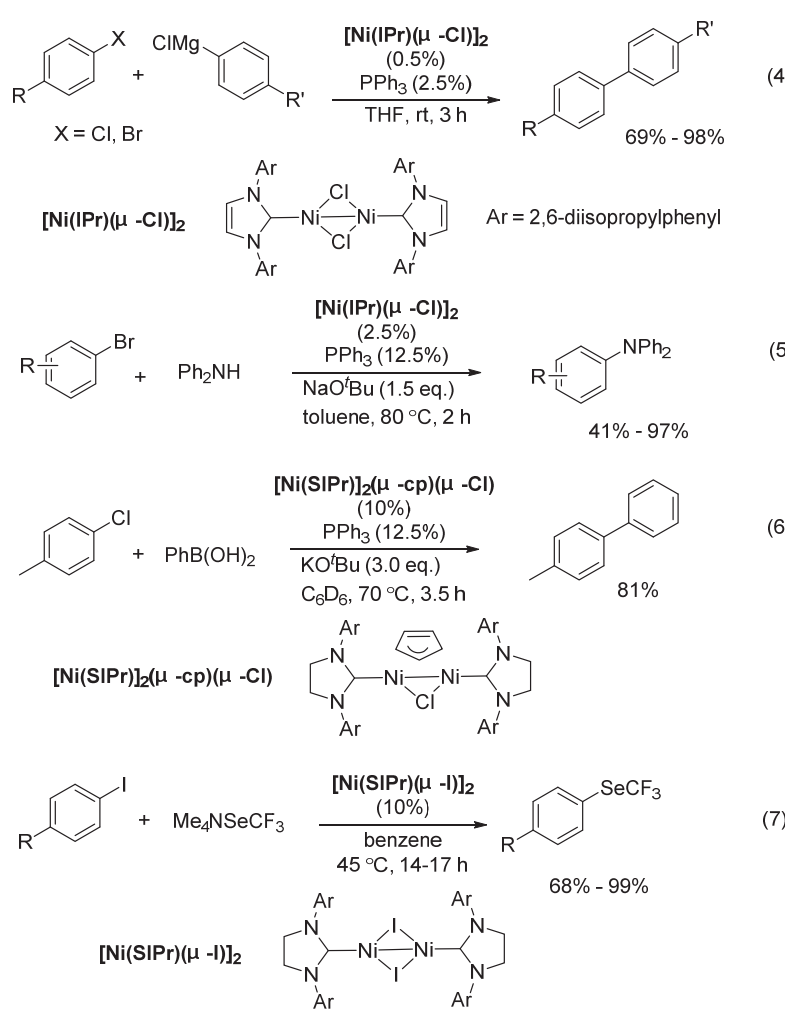

图 20 以卡宾为配体的双核 $\mathrm{Ni}$ 催化的偶联反应 ${ }^{33-37}$

Fig. 20 cross-coupling catalyzed by NHC-bis(Ni) complex ${ }^{33-37}$.

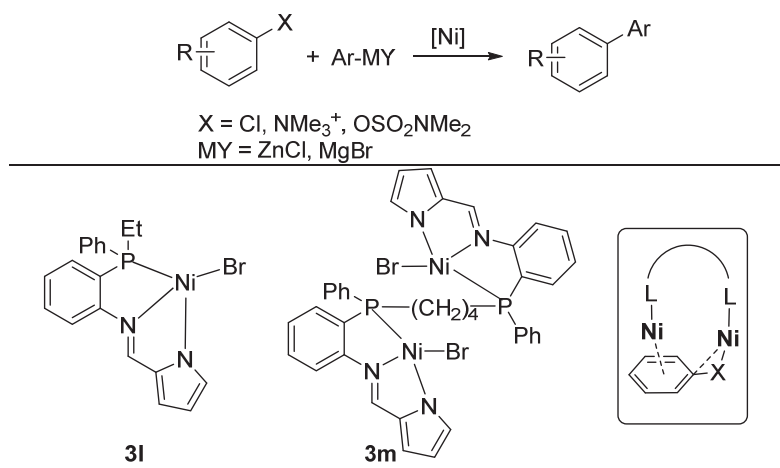

图21 三齿双核Ni催化的偶联反应 ${ }^{38}$

Fig. 21 Cross-copling catalyzed by dinuclear pincer nickel ${ }^{38}$. 


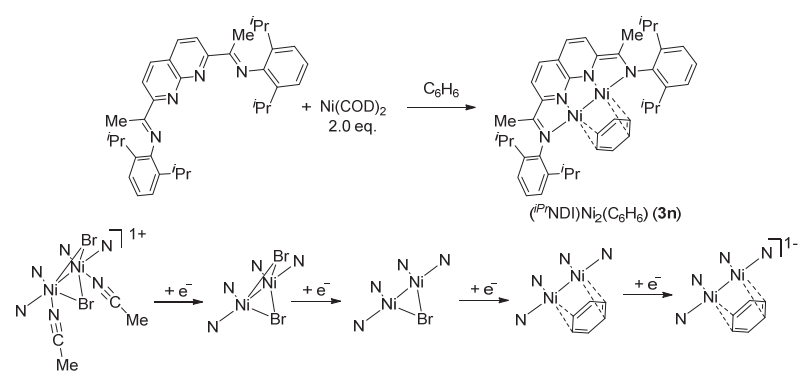

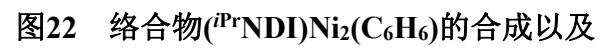
五种氧化还原态 ${ }^{39}$

Fig. 22 The synthesis of $\left({ }^{i{ }^{P r}} \mathrm{NDI}\right) \mathrm{Ni}_{2}\left(\mathrm{C}_{6} \mathrm{H}_{6}\right)$ and five states of oxidation ${ }^{39}$.

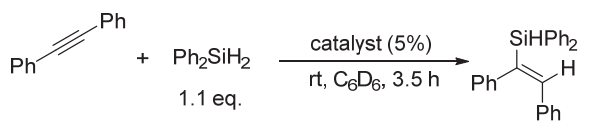
cat. $3 n \quad 93 \%$

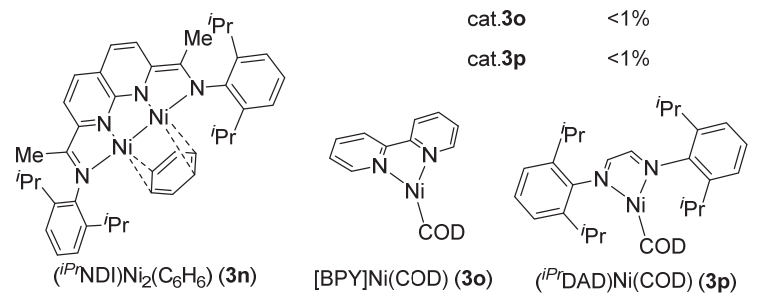

图23 络合物 $3 n$ 催化的炔烃的硅氢化反应 ${ }^{40}$

Fig. 23 Alkyne hydrosilylations catalyzed by complex $3 n^{40}$.

同年, Chirik课题组 ${ }^{41}$ 发现, 以氢桥连, 双亚 胺配体的双核 Ni对催化烯烃的硅氢化反应也具有 很高的活性(图24)。但是该催化剂在催化烯烃硅氢 化反应时, 先转化成单核Ni-H物种后, 才进入催化 循环, 完成催化反应。该类型的催化剂对烯烃的加 氢反应也具有很高的活性 42 。

与此同时, Uyeda课题组 43 发现催化剂 $3 n$ 可以 高效、高选择性的促进炔烃的三聚生成1,2,4-三取 代的芳环类化合物, 产率最高可达到 $90 \%$, 反应选 择性也可以达到20：1以上(图25)。该反应可以兼 容很多类型的官能团, 如醛基、羟基、醚、氟以及 含氧杂环等。而含氮单核 $\mathrm{Ni}$ 络合物如 3o、3p、3q活

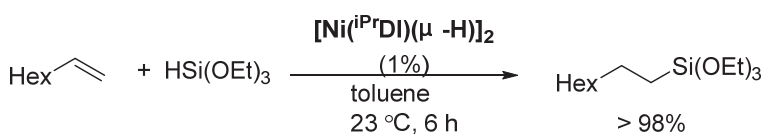

$$
\begin{aligned}
& {\left[\mathrm{Ni}\left({ }^{\mathrm{iPr}} \mathrm{DI}\right)(\boldsymbol{\mu}-\mathrm{H})\right]_{2}}
\end{aligned}
$$

图24 $\left[\mathrm{Ni}\left({ }^{i} \mathrm{PrDI}\right)(\mu-\mathrm{H})\right]_{2}$ 催化的硅氢化反应 ${ }^{41}$

Fig. $24\left[\mathrm{Ni}\left({ }^{i} \operatorname{PrDI}\right)(\mu-\mathrm{H})\right]_{2}$ catalytic hydrosilylations ${ }^{41}$.
性会低很多, 最多只能得到 $14 \%$ 的收率, 而且选择 性很差, 主要得到的是环辛四烯类化合物。

作者通过当量实验对反应机理做了详细的研 究(图26), 在使用大位阻如 $-\mathrm{SiMe}_{3} 、-\mathrm{SiMe}_{2} \mathrm{Ph}$ 取 代的炔烃, 没有得到目标产物。分别得到了 $3 \mathrm{r}$ 与 $3 \mathrm{~s}$ 两种络合物。3r是热力学稳定的, $70{ }^{\circ} \mathrm{C}$ 条件下, $3 \mathrm{r}$ 与 10 当量的三甲基硅乙炔反应 $24 \mathrm{~h}$ 后还是稳定存 在的。但是当把 $3 \mathrm{r}$ 与位阻较小的炔烃反应时, 可以 以 $86 \%$ 的收率得到目标的芳环类产物。 $3 \mathrm{n}$ 与三甲 基硅乙炔反应生成的单炔配位的络合物 $3 \mathrm{~s}$, 在常 温下放置 $24 \mathrm{~h}$ 后会发生歧化反应生成与 $3 r$ 结构类 似的络合物以及络合物 $\mathbf{3 n}$ 。由此可见, 五元环金 属络合物 $3 r$ 是反应的关键中间体。

作者通过理论计算也说明双核金属催化体系 的不同之处：1) 中间体 $\mathbf{3 r}$ 与两个金属中心都有作 用, 这样就限制了金属环的几何形状, 不可能有其
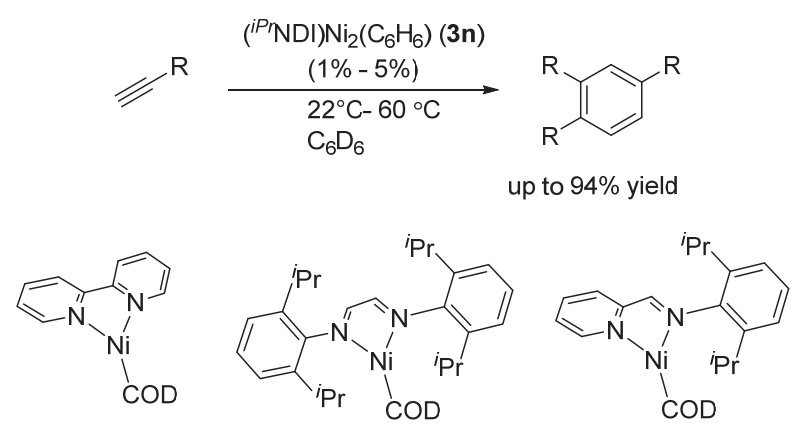

[BPY]Ni(COD) (30) $\quad\left({ }^{\text {iPr }}\right.$ DAD)Ni(COD) (3p) $\quad\left({ }^{i P r} I P\right) N i(C O D)(3 q)$

图25 络合物 $3 n$ 催化炔烃的三聚成环反应 ${ }^{33}$

Fig. 25 Alkyne cyclotrimerizations catalyzed by complex $3 n{ }^{43}$.
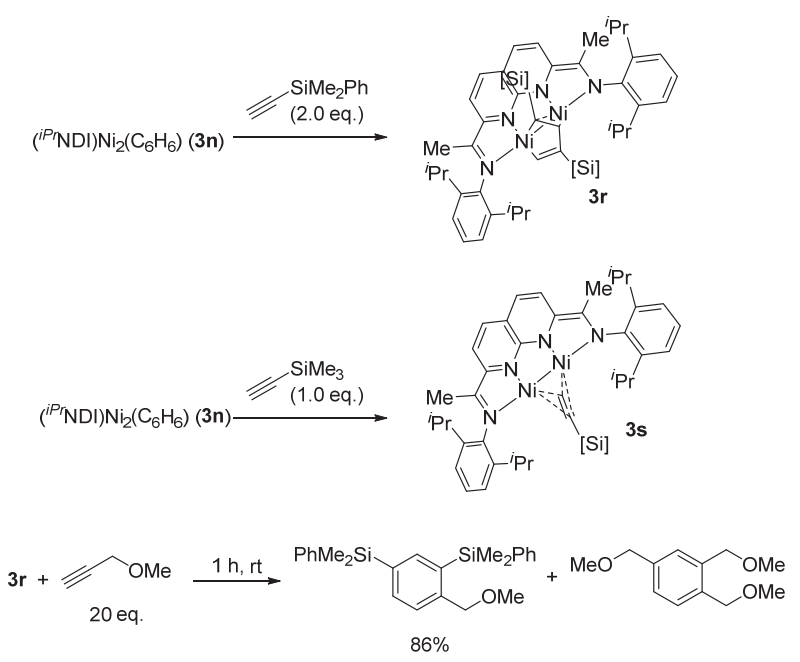

图26 催化剂3n催化炔烃三聚成环反应的机理研究 ${ }^{43}$

Fig. 26 The mechanism of complex 3n catalyzed alkyne cyclotrimerizations ${ }^{43}$. 
他的几何构型。2) 由于金属对丁二烯以及炔烃都 有作用, 有利于 $[4+2]$ 环加成反应。3) 环加成的 选择性受到丁二烯 $\pi$-系统的影响, 导致反应选择 性大大提高。

随后, 该课题组发现这种双核金属 $\mathrm{Ni}$ 催化剂 3n对烯烃的还原环丙烷化也具有很高的催化活性 (图 27, 方程式 8) ${ }^{44}$ 。该反应使用 $\mathrm{CH}_{2} \mathrm{Cl}_{2}$ 做亚甲基 源, $\mathrm{Zn}$ 或者 $\mathrm{ZnEt}_{2}$ 作为还原剂, 反应条件温和, 该 催化体系不仅可以兼容苯乙烯及其衍生物, 对链 状烯烃以及环状烯烃都可以很好的兼容, 各种活 性较高的官能团如羰基、酰胺、酯等都能在该体系 中兼容。该催化剂对催化还原亚乙烯基转移反应 也具有很高的活性(图27, 方程式9) ${ }^{45}$ 。

降冰片二烯的碳一碳 $\sigma$ 键较为情性的, 通常不 容易发生化学转化。2017年, 该课题组 ${ }^{46}$ 发现 $3 n$ 可 以通过对 $\mathrm{C}-\mathrm{C}$ 键的氧化加成, 实现降冰片二烯 $\mathrm{C}-\mathrm{C}$ 的断键(图28)。作者通过当量实验以及理论 计算发现, 该氧化加成是一个迅速放热的过程, 在

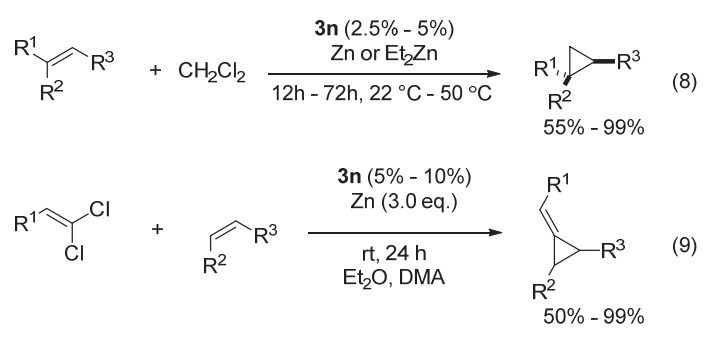

图27 催化剂 $3 n$ 催化的烯烃的还原环丙烷化反应 ${ }^{44,45}$

Fig. 27 Reductive cyclopropanations of alkenes catalyzed by complex $3 n{ }^{44,45}$.

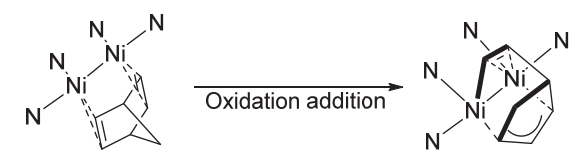

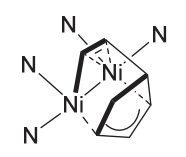

$3 \mathbf{t}$

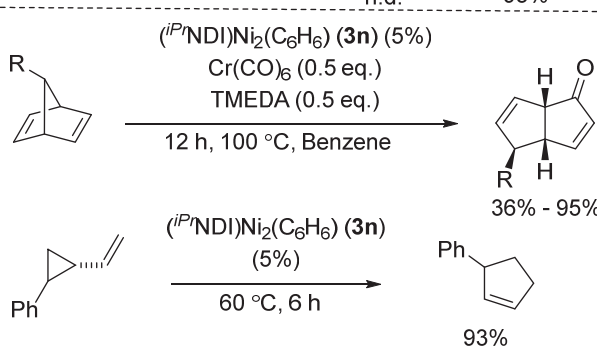

图 28 络合物 $3 n$ 催化 $\mathrm{C}-\mathrm{C}$ 断键反应 ${ }^{46,47}$

Fig. 28 Carbon-carbon bond-breaking reaction catalyzed by complex $3 n{ }^{46,47}$.

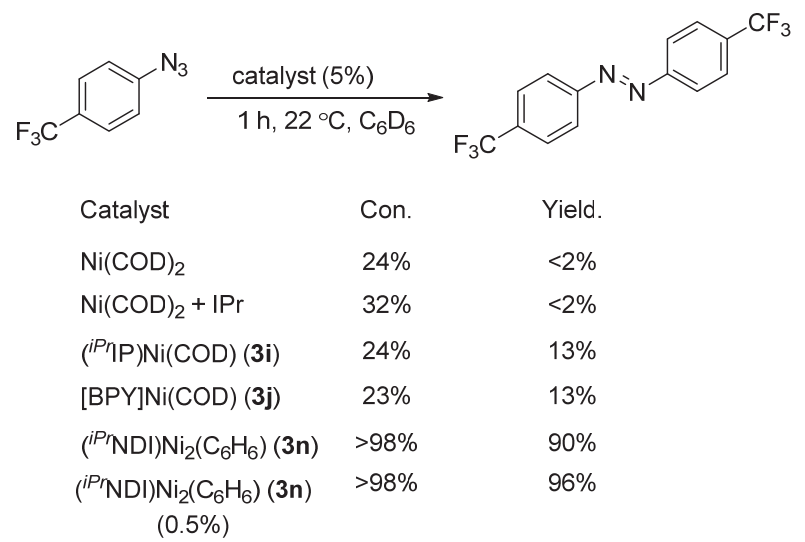

图29 络合物 $3 n$ 催化叠氮化合物的偶联以及与 单核镍催化剂活性比较 48

Fig. 29 The coupling of aryl azides catalyzed by complex $3 \mathrm{n}$ and comparison of the catalytic activation with mononuclear $\mathrm{Ni}$ catalysts ${ }^{48}$.

$30 \min$ 条件下就能完全转化成 $3 t$ (图28, 方程式 $10)$ 。当继续把 $3 \mathrm{t}$ 与 $\mathrm{CO}$ 作用时, 最终以 $93 \%$ 的收率 得到了稠环 $[3,3,0]$ 的产物, 桥连稠环 $[2,3,1]$ 的产物 完全没有, 选择性为 $100 \%$ (图 28, 方程式 11)。最 终作者选用 $\mathrm{Cr}(\mathrm{CO})_{6}$ 为羰基源, TMEDA为添加剂, 实现了催化转化(图28, 方程式12)。随后, 该课题 组还实现了催化剂3n活化环丙类化合物(图28, 方 程式13) ${ }^{47}$ 。

同年，他们使用该催化剂实现了芳基叠氮化 合物的自偶联合成一系列的偶氮化合物(图29) ${ }^{48}$, 该反应体系不需要额外的氧化还原剂, 副产物只 有 $\mathrm{N}_{2}$, 可以兼容对氧化还原条件较为敏感的基团。 作者使用对三氟甲基苯基叠氮化合物的自偶联为 模板反应, 比较了单核 $\mathrm{Ni}$ 催化剂与该双核 $\mathrm{Ni}$ 催化 剂的活性, 研究发现单核 $\mathrm{Ni}$ 催化剂对芳基叠氮化 合物的自偶联反应几乎没有什么活性(转化率在 $23 \%-40 \%$ 之间, 产率 $2 \%-13 \%)$, 而3n几乎可以达 到当量转化, 当催化剂当量降低至 $0.5 \%$ 时, 产率可 达到 $96 \%$ 。随后, 作者进行了机理研究发现, 一分 子的叠氮化合物首先与催化剂 $3 \mathbf{n}$ 形成 $\mu$-NAr的中 间体，然后该中间体再继续与另一分子的叠氮生 成 $\mu-\mathrm{N}_{2} \mathrm{Ar}_{2}$ 络合物, 最后该络合物在溶剂苯的作用 下释放出产物和催化剂, 完成催化循环。最后的解 离过程是该反应的决速步。

\section{5 结论}

金属催化与人们的日常生活息息相关，一直 是人们关注的热点领域。同核铁系双金属络合物 的制备、表征及其性质的研究在近几年得到了迅 猛的发展，尽管目前报道的双核体系金属催化剂 
催化反应的类型还较少, 但仍能看出其相较于传 统的单核金属催化剂的巨大优势。相比较单核金 属催化剂, 双核金属催化剂的催化活性以及反应 选择性都大大提高, 反应条件温和, 操作简单, 而 且还可以实现单核金属催化剂无法完成的催化反 应, 具有重要的应用前景。但是目前同核铁系络合 物在均相催化方面还有一定的局限性, 例如催化 剂当量较高, 机理方面的研究比较滞后, 处于研究 的初级阶段等。因此同核铁系双金属络合物在均 相催化中的应用还需要进一步的开发和应用。

\section{References}

(1) Yu, W. F.; Meng, X. G.; Liu, Y.; Li, X. H. Acta Phys. -Chim. Sin. 2013, 29, 2041. [于卫峰, 孟祥光, 刘芗, 李小红. 物理化学学报, 2013, 29, 2041.] doi: 10.3866/PKU.WHXB201306282

(2) Luo, K. J.; Geng, H.; Zhang, C. Y.; Ni, H. L.; Li, Q. Chin. J. Inorg. Chem. 2017, 33, 405. [骆开均, 耿浩, 张晨阳, 倪海亮, 李权. 无机 化学学报, 2017, 33, 405.] doi: 10.11862/cjic.2017.026

(3) Huang, S. P.; Yuan, Z. Z. Acta Phys. -Chim. Sin. 2009, 25, 1599. [黄斯娉, 袁中直. 物理化学学报, 2009, 25, 1599.] doi: 10.3866/PKU.WHXB20090753

(4) Guo, L. Q.; Shi, X. L.; Ruan, W. J.; Zhang, X. H.; Zhu, Z. A. Acta Phys. -Chim. Sin. 2010, 26, 1195. [郭莉芹, 史秀丽, 阮文娟, 张晓 红, 朱志昂. 物理化学学报, 2010, 26, 1195.] doi: 10.3866/PKU.WHXB20100336

(5) Liu, H.; Zang, N.; Zhao, F. Y.; Liu, K.; Li, Y.; Ruan, W. J. Acta Phys. -Chim. Sin. 2014, 30, 1801. [刘欢, 㶓娜, 赵芳瑶, 刘坤, 李悦, 阮文娟. 物理化学学报, 2014, 30, 1801.] doi: 10.3866/PKU.WHXB201407171

(6) Liu, F. Q.; Zhao, J.; Zhang, D.; Duan, X. Q.; Wang, L.; Deng, Y. Y.; Li, W. H. Chin. J. Inorg. Chem. 2015, 31, 1402. [刘法谦, 赵杰, 张东, 段小群, 王磊, 邓月义, 李伟华. 无机化学学报, 2015, 31, 1402.] doi: $10.11862 /$ cjic. 2015.166

(7) Behlen, M. J.; Zhou, Y. Y.; Steiman, T. J.; Pal, S.; Hartline, D. R.; Zeller, M.; Uyeda, C. Dalton Trans. 2017, 46, 5493. doi: $10.1039 / \mathrm{c} 6 \mathrm{dt} 04465 \mathrm{~d}$

(8) Gavrilova, A. L.; Bosnich, B. Chem. Rev. 2004, 104, 349. doi: $10.1021 / \mathrm{cr} 020604 \mathrm{~g}$

(9) Do, L. H.; Xue, G.; Que, L., Jr.; Lippard, S. J. Inorg. Chem. 2012, 51, 2393. doi: $10.1021 /$ ic $202379 b$

(10) Ohki, Y.; Hatanaka, T.; Tatsumi, K. J. Am. Chem. Soc. 2008, 130, 17174. doi: 10.1021/ja8063028

(11) Zhang, F.; Song, H.; Zhuang, X.; Tung, C. H.; Wang, W. J. Am. Chem. Soc. 2017, 139, 17775. doi: 10.1021/jacs.7b11416

(12) Obligacion, J. V.; Chirik, P. J. Org. Lett. 2013, 15, 2680. doi: $10.1021 / \mathrm{ol} 400990 \mathrm{u}$
(13) Iovan, D. A.; Betley, T. A. J. Am. Chem. Soc. 2016, 138, 1983. doi: $10.1021 /$ jacs.5b12582

(14) Tong, P.; Yang, D.; Li, Y.; Wang, B.; Qu, J. Organometallics 2015, 34 , 3571. doi: 10.1021/acs.organomet.5b00387

(15) Wilkinson, E. C.; Dong, Y.; Que, L. J. Am. Chem. Soc. 1994, 116, 8394. doi: $10.1021 / \mathrm{ja} 00097 \mathrm{a} 068$

(16) Mo, Z.; Zhang, Q.; Deng, L. Organometallics 2012, 31, 6518. doi: $10.1021 / \mathrm{om} 300722 \mathrm{~g}$

(17) Chen, Z.; Furutachi, M.; Kato, Y.; Matsunaga, S.; Shibasaki, M. Angew. Chem. Int. Ed. 2009, 48, 2218. doi: 10.1002/anie.200805967

(18) Gao, J.; Woolley, F. R.; Zingaro, R. A. Org. Biomol. Chem. 2005, 3, 2126. doi: $10.1039 / \mathrm{b} 503971 \mathrm{a}$

(19) Kember, M. R.; White, A. J. P.; Williams, C. K. Macromolecules 2010, 43, 2291. doi: 10.1021/ma902582m

(20) Yu, C. Y.; Chuang, H. J.; Ko, B. T. Catal. Sci. Technol. 2016, 6, 1779. doi: $10.1039 / \mathrm{c} 5 \mathrm{cy} 01290 \mathrm{~b}$

(21) Lin, P. -M.; Chang, C. -H.; Chuang, H. -J.; Liu, C. -T.; Ko, B. -T.; Lin, C. -C. ChemCatChem 2016, 8, 984. doi: $10.1002 /$ cctc. 201501280

(22) Chen, Z.; Morimoto, H.; Matsunaga, S.; Shibasaki, M. J. Am. Chem. Soc. 2008, 130, 2170. doi: 10.1021/ja710398q

(23) Chen, Z.; Yakura, K.; Matsunaga, S.; Shibasaki, M. Org. Lett. 2008, 10, 3239. doi: 10.1021/o1800965t

(24) Shepherd, N. E.; Tanabe, H.; Xu Y.; Matsunaga, S.; Shibasaki, M. J. Am. Chem. Soc. 2010, 132, 3666. doi: 10.1021/ja1002636

(25) Mouri, S.; Chen, Z.; Matsunaga, S.; Shibasaki, M. Chem. Commun. 2009, 5138. doi: 10.1039/b912380f

(26) Shibasaki, M.; Matsunaga, S.; Kato, Y.; Chen, Z. Synlett 2009, 2009, 1635. doi: 10.1055/s-0029-1217192

(27) Mitsunuma, H.; Matsunaga, S. Chem. Commun. 2011, 47, 469. doi: $10.1039 / \mathrm{c} 0 \mathrm{cc} 02152 \mathrm{k}$

(28) Xu, Y.; Matsunaga, S.; Shibasaki, M. Org. Lett. 2010, 12, 3246. doi: 10.1021/ol101185p

(29) Gopinath, P.; Watanabe, T.; Shibasaki, M. Org. Lett. 2012, 14, 1358. doi: $10.1021 / 013002078$

(30) Zhang, S.; Deng, P.; Zhou, J.; Liu, M.; Liang, G.; Xiong, Y.; Zhou, H. Chem. Commun. 2017, 53, 12914. doi: 10.1039/c7cc06468c

(31) Dible, B. R.; Sigman, M. S. J. Am. Chem. Soc. 2003, 125, 872. doi: $10.1021 / \mathrm{ja} 0286876$

(32) Laskowski, C. A.; Hillhouse, G. L. Organometallics 2009, 28, 6114. doi: $10.1021 / 0 m 900783 \mathrm{u}$

(33) Miyazaki, S.; Koga, Y.; Matsumoto, T.; Matsubara, K. Chem. Commun. 2010, 46, 1932. doi: 10.1039/b924716e

(34) Matsubara, K.; Yamamoto, H.; Miyazaki, S.; Inatomi, T.; Nonaka, K.; Koga, Y.; Yamada, Y.; Veiros, L. F.; Kirchner, K. Organometallics 2016, 36, 255. doi: 10.1021/acs.organomet.6b00451 
(35) Nagao, S.; Matsumoto, T.; Koga, Y.; Matsubara, K. Chem. Lett. 2011, 40, 1036. doi: 10.1246/c1.2011.1036

(36) Wu, J.; Nova, A.; Balcells, D.; Brudvig, G. W.; Dai, W.; Guard, L. M.; Hazari, N.; Lin, P. H.; Pokhrel, R.; Takase, M. K. Chem. Eur. J. 2014, 20, 5327. doi: 10.1002/chem.201305021

(37) Durr, A. B.; Fisher, H. C.; Kalvet, I.; Truong, K. N.; Schoenebeck, F. Angew. Chem. Int. Ed. 2017, 56, 13431. doi: 10.1002/anie.201706423

(38) Yang, X.; Wang, Z. -X. Organometallics 2014, 33, 5863. doi: $10.1021 / \mathrm{om} 500452 \mathrm{c}$

(39) Zhou, Y. Y.; Hartline, D. R.; Steiman, T. J.; Fanwick, P. E.; Uyeda, C. Inorg. Chem. 2014, 53, 11770. doi: 10.1021/ic5020785

(40) Steiman, T. J.; Uyeda, C. J. Am. Chem. Soc. 2015, 137, 6104. doi: $10.1021 /$ jacs.5b03092

(41) Pappas, I.; Treacy, S.; Chirik, P. J. ACS Catal. 2016, 6, 4105. doi: $10.1021 /$ acscatal.6b01134
(42) Léonard, N. G.; Chirik, P. J. ACS Catal. 2017, 8, 342. doi: 10.1021/acscatal.7b03909

(43) Pal, S.; Uyeda, C. J. Am. Chem. Soc. 2015, 137, 8042. doi: 10.1021/jacs.5b04990

(44) Zhou, Y. -Y.; Uyeda, C. Angew. Chem. Int. Ed. 2016, 55, 3171. doi: 10.1002/anie.201511271

(45) Pal, S.; Zhou, Y. Y.; Uyeda, C. J. Am. Chem. Soc. 2017, 139, 11686. doi: $10.1021 /$ jacs.7b05901

(46) Hartline, D. R.; Zeller, M.; Uyeda, C. J. Am. Chem. Soc. 2017, 139, 13672. doi: $10.1021 /$ jacs. 7 b08691

(47) Rounds, H. R.; Zeller, M.; Uyeda, C. Organometallics 2018, 37, 545. doi: 10.1021/acs.organomet.7b00862

(48) Powers, I. G.; Andjaba, J. M.; Luo, X.; Mei, J.; Uyeda, C. J. Am. Chem. Soc. 2018, 140, 4110. doi: 10.1021/jacs.8b00503 